\title{
Interdisciplinary safety analysis of complex socio-technological systems based on the Functional Resonance Accident Model: an application to railway traffic supervision
}

\author{
Fabien Belmonte ${ }^{\mathrm{a}}$, Walter Schön ${ }^{\mathrm{b}}$, Laurent Heurley ${ }^{\mathrm{c}}$, Robert Capel $^{\mathrm{a}}$ \\ ${ }^{a}$ Alstom Transport \\ 48 rue Albert Dhalenne \\ 93482 Saint-Ouen cedex, France \\ ${ }^{b}$ Université de Technologie de Compiègne \\ Laboratoire Heudiasyc \\ Centre de Recherches de Royallieu \\ BP20529 \\ 60205 Compiègne cedex, France \\ ${ }^{c}$ Université de Picardie Jules Verne \\ Équipe Cognition, Langage, Émotion et Acquisition (CLEA) \\ EA 4296, UFR de Philosophie, Sciences Humaines et Sociales \\ Chemin du Thil, 80025 Amiens, Cedex 1.
}

\begin{abstract}
This paper presents an application of Functional Resonance Accident Models (FRAM) for the safety analysis of complex socio-technological systems, i.e. systems which include not only technological, but also human and organizational components. The supervision of certain industrial domains provides a good example of such systems, because although more and more actions for piloting installations are now automatized, there always remains a decision level (at least in the management of degraded modes) involving human behavior and organizations. The field of application of the study presented here is railway traffic supervision, using modern Automatic Train Supervision (ATS) systems. Examples taken from railway traffic supervision
\end{abstract}


illustrate the principal advantage of FRAM in comparison to classical safety analysis models, i.e. their ability to take into account technical as well as human and organizational aspects within a single model, thus allowing a true multidisciplinary cooperation between specialists from the different domains involved.

A FRAM analysis is used to interpret experimental results obtained from a real ATS system linked to a railway simulator that places operators (experimental subjects) in simulated situations involving incidents. The first results show a significant dispersion in performances among different operators when detecting incidents. Some subsequent work in progress aims to make these "performance conditions" more homogeneous, mainly by ergonomic modifications. It is clear that the current Human-Machine Interface (HMI) in ATS systems (a legacy of past technologies that used LED displays) has reached its limits and needs to be improved, for example, by highlighting the most pertinent information for a given situation (and, conversely, by removing irrelevant information likely to distract operators).

Keywords:

Automatic Train Supervision (ATS), Human-Machine Interface (HMI), Human-Machine System (HMS), Human Reliability, Functional Resonance Accident Model (FRAM), Simulated environment, Experiments.

\section{Introduction}

Industrial risk management has become a major challenge for our society. Born out of the need to control new hazards that have accompanied technological advances (especially since the second half of the $20^{\text {th }}$ century), 
industrial risk management was first formalized in the nineteen-sixties with the emergence of reliability and maintainability theory [1]. The designers of safety-critical systems subsequently incorporated this formalism into the standard techniques of systems engineering [2]. These techniques have now been normalized for particular domains of activity. See for example [3] for programmable electronics, or $[4,5,6]$ for the specific domain of railway signaling. Such developments did not, however, explicitly include any reference to human factors. Nevertheless, in the military domain the central role of the human operator in the operation of safety-critical systems was quickly recognized. Norbert Wiener's Cybernetics [7], for example, drew on observations of an anti-aircraft defence operator and a perceived analogy between the mechanical laws governing the physical evolution of the system and the operator's behavior. A number of studies aiming to improve working conditions have been successfully conducted and have contributed to reducing risks. However, establishing a direct link between risk management and the social and human sciences remains somewhat elusive. The methodological framework for risk management now includes specific tools for evaluating human reliability, and more recently organizational reliability. The first methods to be developed used the formal framework of reliability, which meant approximating human behavior to machine behavior. This approach was strongly criticized by the scientific community. Dougherty [8] and Hollnagel [9] provide a summary of this criticism. This first generation of methods also initiated a debate about the nature of human error [10]. In the following years a second generation of methods emerged, taking into account human and social factors in a more detailed manner and drawing upon developments 
in the human and social sciences $[9,11,12,13]$; see [14] for a review of the literature.

This paper deals with industrial risks assessment in complex socio-technical systems comprising a technical level (hardware and software), a human level (maintenance agents, operators, designers) and an organizational level (a set of rules and interactions governing the different actions that can be performed on the system). Each one of these components could be addressed separately by adopting either a machine-centered approach (or technicalcentered approach) or a human-centered approach. Instead, we propose to adopt a Human-Machine System (HMS) approach that considers these separate levels in a systemic way [15].

To illustrate our approach we present an interdisciplinary study we have conducted to evaluate the impact of automation in a railway traffic supervision subsystem on the overall safety of the railway system. The methodology used in this study involves carrying out the risk analysis as recommended by each framework, and applying a complementary analysis to take into account the human and organizational levels in a more satisfactory manner than the classical approach to the safety of technological systems. This complementary approach must establish a referential common to the three scientific domains involved in the complex socio-technical systems.

In the first part of the paper, we present briefly the HMS approach we adopted. The second part uses the theory of accident modeling to demonstrate the need for a systemic approach in evaluating the human and organizational levels. The Functional Resonance Accident Model (FRAM), developed by E. Hollnagel [16] to analyze accidents, uses this approach, where 
the three components (technical, human, organizational) are present simultaneously and interact continuously. This method and its application in the railway field are presented in the last two sections: we begin with the detailed presentation of a safety procedure for protecting a zone of work on a railway track where the different levels of human activity are strongly impacted, after which we examine a further case study involving traffic monitoring and incident detection by operators. In this second case the corresponding FRAM network is calibrated using experimental results obtained from real operators during simulations. Used as a complement to the classical approach, the FRAM method can provide a more detailed insight into human and organizational events, allowing qualitative results to be refined. We conclude the paper with some perspectives for future work: the main objectives of further work will be to evaluate the likely benefits of improving Human-Machine Interfaces (HMI) in ATS systems as regards the detection of incidents by human operators, and possibly to seek to derive quantitative analysis from qualitative analysis using belief function approaches.

This work is supported by the French State and the "Picardie" Region as part of the "Human, Technologies and Complex Systems" research programme. It was the subject of a PhD. thesis defended in 2008 [17].

\section{Machine-centered vs. Human-Machine System approach}

The present paper adopts an HMS approach rather than a machinecentered approach.

In terms of the discipline, the machine-centered approach corresponds to an engineering approach, the concepts and tools of industrial risk manage- 
ment being based on standard techniques of dependability, using the mathematical formalism of reliability and maintainability developed by Barlow and Proschan [1]. This formalism contains a bi-modal model of the system's components with only two possible states (functioning / failure) for each component. Other methods, such as Fault Tree Analysis, are able to deal with multimodal problems [18] i.e. taking more than one failure mode into account. In all cases the system is characterized by a characteristic vector giving the state of each of its components. The approach involves determining the system's state as a function of this characteristic vector. Rigorous methods, using inductive or deductive reasoning, are employed to this end. The methodology is based on a systematic exploration of hazardous events or components' failure modes. The methods developed by dependability engineers provide the tools needed to explain the failures at the system's different levels that result in accidents (see [19] for a detailed presentation of these methods).

However, this machine-centered approach appeared to be insufficient to improve the reliability of complex automated systems such as nuclear reactors or transportation systems. It became clear that human operators are a key component in reliability and safety of such systems. For example, Rasmussen 1969 cited by Vicente [20] points out that one of the conclusions of an analysis of 129 industrial accidents in the nuclear and air transportation domains conducted by the cross-disciplinary research team of the RIS $\varnothing$ group was that "accidents initiated by human maloperation amount to roughly three quarters of the total number of reported cases" [20] page 11.

For this reason, from the beginning of the 1960s some researchers began 
to shift from a machine-centered approach to an HMS approach [20, 21]. From the start, one of the main problems faced by researchers and designers was analyzing and understanding the impact of interface design on operators' cognitive processes and performances when interacting with complex systems (e.g., in nuclear control rooms) in order to improve the way information was presented to operators. Instead of finding answers to their questions in the psychological literature they found a gap, and in an attempt to fill this gap were led to carry out research and develop new models and methods adapted to HMS. They used concepts, approximate models of cognition [22] and methods developed in cognitive psychology and in cognitive science.

Nowadays, the HMS approach is well represented in three fields which emerged at the end of the 1970s and at the beginning of the 1980s. These fields, whose frontiers are still not clearly identified, are cognitive engineering, cognitive systems engineering and cognitive ergonomics [23, 15]. Cognitive engineering can be defined as "an applied cognitive science that draws on the knowledge and techniques of cognitive psychology and related disciplines to provide the foundation for principle-driven design of person-machine systems". [23] p. 415. Cognitive systems engineering is a variant of cognitive engineering in which work, humans and technology are considered as a joint cognitive system [24]. It has recently been defined as "an approach to the design of technology, training, and processes intended to manage cognitive complexity in sociotechnical systems." [25] p. 3. Cognitive ergonomics adopts a similar approach to the two others, but having emerged in the context of human-computer interactions cognitive ergonomics focuses more closely on the role of the human in HMS, whereas cognitive engineering and cogni- 
tive systems engineering place greater emphasis on the role of the machine $[26,15]$.

These fields both consider that the psychological functioning of operators (improperly referred to by the terms "cognitive" or "cognition" [21]) is a crucial part of the overall reliability and safety of the rest of the system in/with which they interact, and consequently must be taken into account and incorporated in the design process of complex systems. This position is well illustrated by Hollnagel and Woods who consider that "the costs and consequences of ignoring the cognitive functions of Man Machine Systems are noted in technological failures daily (we need only mention the Three Mile Island accident)" [21] p. 585. Certain concepts, as well as theories about the architecture and functioning of human cognition that were initially elaborated in the context of laboratory research (principally in an informationprocessing perspective), and also methods taken from cognitive psychology and cognitive science, have been used and adapted for practical needs by these three fields $[27,28]$.

As it will appear in the following sections, our research is situated at the meeting point of these different fields.

\section{Accident theory models}

The analytical method used in dependability analysis represents accidents as successions of events in which safety appears as a property of the system. Resilience engineering [29], in a systemic framework, goes beyond this and defines safety as an emergent system phenomenon, rather than as a property. 


\subsection{Accident models}

To facilitate this approach a classification of accidents into three categories has been proposed [16]: sequential, epidemiological and systemic.

In the sequential model, an accident is explained as a succession of events linked together by a cause-to-effect relationship. In Reason's [30] epidemiological model an accident is the result of passive failures, introduced by latent conditions whose effect is not immediate, but becomes manifest when a particular function or system component is called upon. Finally, the systemic model introduced by Woods [31], Leveson [19] and Hollnagel [16] describes an accident as the result of complex interactions between the different com-

ponents of the system. An accident is the consequence of a coincidence of events, rather than of a deterministic succession of events [16].

\subsection{Methodological referential}

Dependability theories have not yet developed methods for evaluating the dynamics of accidents relative to all three models listed above. Only the first two models are addressed in the methodological framework. The main methods derive from two approaches [2]:

- inductive approach (from causes to consequences: methods include Event-Trees, Failure Modes Effects and Criticality Analysis);

- deductive approach (from consequences to causes: methods include Fault Trees).

Several methods based on the same theoretical background have been developed in order to address human factors and/or organizational risk analysis; see [32] for a global overview of these methods. 


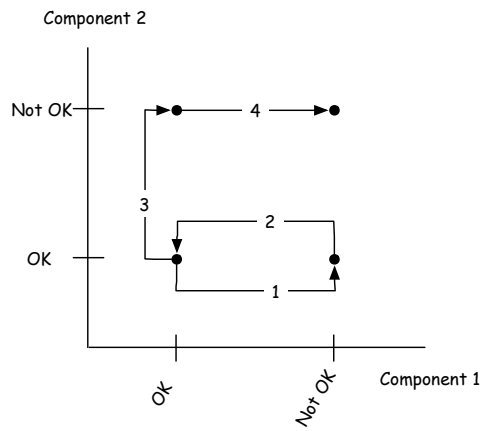

Figure 1: Example of discrete scenario space

All these methods are widely used in various industrial domains and recommended by the normative referential. Each of them creates a representation of the system using the state of its components, which can be bi-modal (functioning / failure) or multi-modal (several degraded modes and failure modes). These methods enable the system's state to be inferred from its state vector (that is to say the values of the bi-modal or multi-modal variables defining the state of system components), and make it possible to plot the evolution of system state via a state diagram. As an example, Figure 1 represents a system composed of two components with bi-modal states, which leads to four states for the system. The path illustrated in the figure shows a scenario for the evolution of system state in four steps. This representation of accidents fulfils the requirement for the safety of a technical system to be demonstrable, provided that it can be shown that the set of pre-established failure scenarios is exhaustive (in terms of the severity of the consequences). It is, however, too restrictive when dealing with events involving humans and organizations. Considering human beings as bi-modal components (or as components with only a few clearly identified failure modes) is obviously 
too restrictive and does not correspond to the models to be found cognitive psychology. In an attempt to go beyond this overly simplistic representation, systemic models have focused on system functions instead of on system components. The purpose is to be able to represent socio-technical system dynamics, in a continuum based on measurements of the effectiveness of functions performed by the system. Figure 2 presents a continuous path representing the state of a system performing three functions. The difficulty is to find an appropriate measurement of the effectiveness of each function.

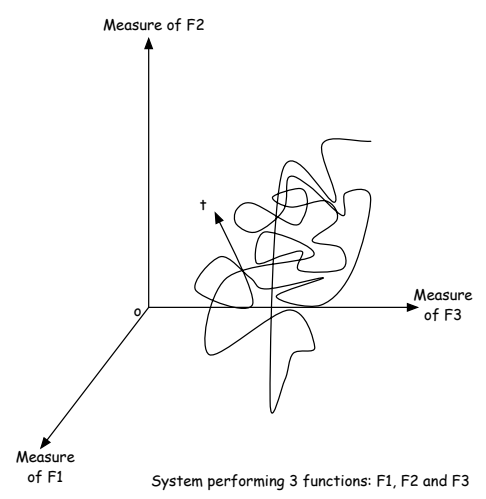

Figure 2: Example of continuous scenario space

\subsection{A common referential: systems analysis}

The major advantage of a systemic approach is a more complete integration of "human factor oriented" studies on the one hand, and "technical safety oriented" studies on the other. This is achieved largely through the use of a common methodological referential. Woods and Hollnagel [33, 34, 35] see socio-technical systems as cognitive systems, referring to them as "Joint Cognitive Systems". These authors have developed a new branch of engineering 
for human-machine interaction which has become known as Cognitive Systems Engineering (CSE) [36, 37]. Human-machine systems have traditionally been analyzed using separate approaches for the human and technical components, followed by a complementary study of interactions. For the creators of CSE, this reductive approach is inadequate, and should be replaced by a representation of the complete system including human operators and machines. The common denominator is given by the cognitive dimension of this overall system.

However, a representation of the complete system will not in itself improve the system's safety. Internal relationships within the system do not have identical weight, nor do they have identical consequences for safety. For

Bieder [13], a systemic representation is not sufficient by itself to yield an integrated approach for the safety of complex socio-technical systems. A joint endeavor bridging the divide between dependability engineering and the human and social sciences is also needed. However, performing safety studies by systemic methods is, in our view, a fundamental complement to classical safety analysis. It is in this perspective that the Functional Resonance Analysis Method was put forward.

\section{The Functional Resonance Analysis Method}

The FRAM method, developed by E. Hollnagel [16], describes the sociotechnical system in terms of its functions and its activities rather than in terms of its structure. The aim of the FRAM method is to represent the system's dynamics by modeling the non-linear interactions that are part of the system, and through a novel representation of the performance of 
functions and activities. The dependency model is based on the concept of functional resonance, which comes from undulatory physics and can be seen as a metaphor for stochastic resonance. The stochastic resonance principle involves superimposing noise, in non-linear systems, on a low amplitude signal that is hardly detectable. Adding the noise creates a "resonance" with the low amplitude signal that makes it detectable. Hollnagel here makes use of the concept of stochastic resonance (more often used to explain the appearance of order in a system) in order to explain the occurrence of accidents. He carries out this transfer from physics to safety analysis by using the variability of performance of operational units within a socio-technical system. Traditionally, functions represent technical system operations and activities represent human/organizational operations; here the two concepts are merged in the concept of "operations". Within a socio-technical system operational units might be technical systems, humans or organizations. In the view of [16], variability of performance in a technical system is related to imperfections arising out of the processes of design and production, and to non-exhaustive specifications of working conditions (effects due to the environment and to non-expected inputs). Variability of performance in humans and organizations comes from their capacity to adapt themselves to working conditions and from the irregularity in activities (perception, cognition, action and communication). The parallel with stochastic resonance can be explained by the stochastic nature of the variability of performance of operational units within the socio-technical system, considered as a nonlinear system. In addition, Hollnagel uses the superposition of the signal as a functional dependency model between system's operational units. The low 
amplitude signal corresponds to the variability of performance of a particular operational unit. This variability is a "low amplitude signal" insofar as deviations in performances (considered individually and not in the global system context) have little or no impact on the global system performance and on safety. The "noise" signal creating the resonance corresponds to the variability of performance of the rest of the system. The low amplitude signal may therefore be the variability of performance of any operational unit in the system, and consequently the "noise" corresponds to the aggregation of the variability of performance of all other operational units (including the environment). Hollnagel calls this phenomenon "functional resonance".

Examining the potentiality of accidents using the FRAM method means examining the operation of a particular system in three separate steps that we outline below.

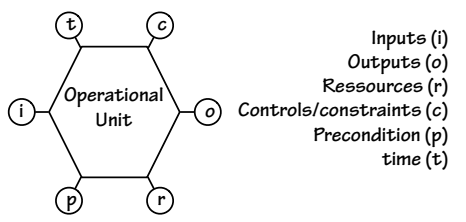

Figure 3: Hexagram

The first step is a formal breakdown of system operation into elementary operational units, each of which has six attributes (see Figure 3). These attributes serve as connectors between elementary operational units:

- Inputs (i): input(s) of the operational unit;

- Outputs (o): output(s) of the operational unit; 
- Resource (r): resource(s) required for the processing performed by the operational unit;

- Times (t): time required for the processing performed by the operational unit;

- Control (c): control(s) and constraint(s) governing processing by the operational unit (exceptions, procedures, methods, etc.);

- Precondition (p): conditions that must be satisfied for the operational unit to function correctly.

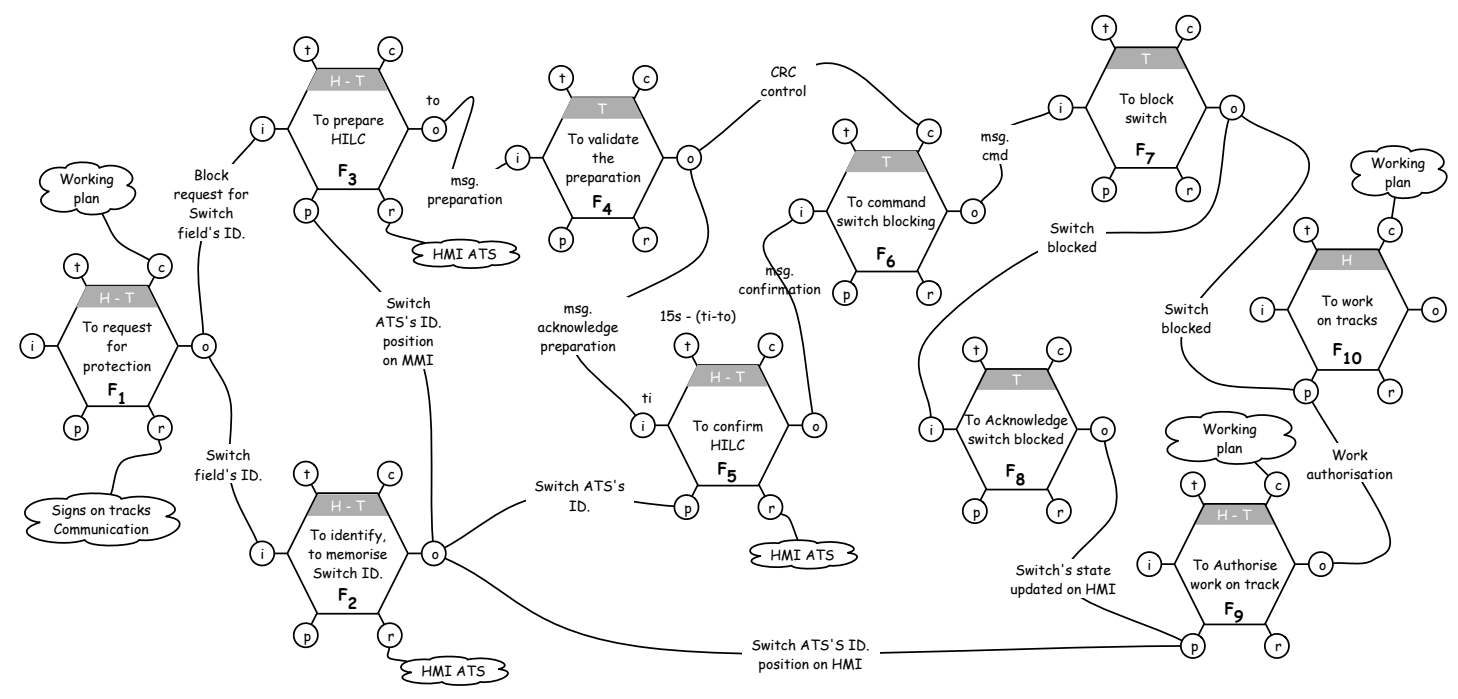

Figure 4: FRAM network model

The second step is determining the potential variability of each operational unit. FRAM classifies operational units into three categories: Human $(\mathrm{H})$, Technical $(\mathrm{T})$ and Organizational $(\mathrm{O})$. The operational unit's potential variability is determined by the respective weights of eleven common 
performance conditions (CPC), acting as context factors on the operational unit, according to its category. The CPC used in FRAM are based on Hollnagel's CREAM (Cognitive Reliability and Error Analysis Method) method for studying human reliability; see [9] for a detailed presentation.

The CPC are shown in Table 1, according to operational unit category. These context factors can have positive or negative impacts on performance.

\begin{tabular}{lc}
\hline CPC & Category \\
\hline Resource availability & $\mathrm{H}-\mathrm{T}$ \\
Training and experience & $\mathrm{H}$ \\
Quality of communications & $\mathrm{H}-\mathrm{T}$ \\
Quality of human-machine interfaces & $\mathrm{T}$ \\
Accessibility and availability of meth- & $\mathrm{H}$ \\
ods and procedures & \\
Working conditions & $\mathrm{H}-\mathrm{T}$ \\
Number of simultaneous objectives & $\mathrm{H}-\mathrm{O}$ \\
Time available & $\mathrm{H}$ \\
Circadian rhythm & $\mathrm{H}$ \\
Quality of team collaboration & $\mathrm{H}$ \\
Quality of organizational support & $\mathrm{O}$ \\
\hline
\end{tabular}

Table 1: Common Performance Conditions (CPC)

The quality of each CPC takes one of three possible values: (1) stable or variable but adequate, (2) stable or variable but inadequate, (3) unpredictable. The aim is to determine the set of applicable CPCs for each operational unit and to evaluate their quality. As a general rule, where a CPC is stable or variable but adequate the corresponding variability of performance is low, and where the CPC is stable or variable but inadequate, variability of performance is high. Finally, if a CPC is unpredictable, the corresponding 
variability of performance is very high.

The third step establishes dependencies between operational units. This is easily performed by inserting correspondence attributes assigned in the first step. Graphically, this means connecting the inputs and outputs of each unit, represented by its hexagon, to the outputs and inputs of other units, thus constituting what is termed a "FRAM network": Figure 4 shows an example of a FRAM network constructed in this way (this network represents the railway case study presented in the following section, for protecting maintenance teams on tracks, in its nominal mode). This network enables information flows during the normal execution of an operation to be represented formally. The labels $\mathrm{H}, \mathrm{T}$ and $\mathrm{O}$ in the header part of a unit's hexagon indicate the category of entities participating in the operational unit. The network is then used to search for negative functional resonances which may affect the correct sequence of operational units and to detect the propagation of such resonances through the system.

The following section presents a case study of the use of FRAM in the domain of railway safety.

\section{Application to railway traffic supervision}

\subsection{Railway supervision context}

In the past, the safety of railway traffic was almost entirely dependent on actions by humans (mechanical signals were manually manipulated by agents). Now railway systems are widely automated:

- On most lines, trains are automatically and safely detected inside fixed zones known as "blocks" (very few lines currently use "moving blocks" 
whose positions depend on the movements of trains). Safe detection means that no single failure or plausible combination of failures can lead to the non-detection of a train (consequently failures usually lead to false detections);

- This automatic train detection is used to operate signals which ensure that trains only enter blocks that are not already occupied. This function is called "block logic" or "block system";

- To accommodate trains going in several directions, a railway network includes branch lines and junctions that rely on "switches" or "points". The state of the switches is safely controlled;

- Automatic train detection, switch control and signals are used to allow a train to travel on a "route" (a section of track between two switches) only if no trains are simultaneously allowed to travel on a conflicting (i.e. opposite direction, convergent, sequent, etc.) route. This function is called "interlocking";

- If a driver fails to respect a signal, this will be detected and result in the train being stopped. This function is known as "Automatic Train Protection" (ATP);

- On some urban lines trains are driven automatically, and in certain cases no driver is present in the cab. This function, known as "Automatic Train Operation" (ATO) is not considered safety critical (since safety is ensured by ATP function); 
- Finally, the control and monitoring of traffic is more and more centralized in "Operating and Control Centers" (OCC). This function, known Automatic Train Supervision (ATS) is the framework for the present paper.

The ATS function has evolved considerably in recent years: it now makes wide use of computer systems to perform functions like "automatic route setting" (routes are automatically set for trains using predefined "timetables" or "time schedules" where each train's mission is defined). Therefore, in a completely nominal situation the human operator's role is restricted to monitoring (see [38] for a detailed description of "ironies of automation"). However, human interventions remain necessary to recover from unavoidable incidents which make some trains late, make some sections of track unavailable, and so on. In such cases, human operators will use ATS to modify the schedule, to manually set routes, etc. Although this might seem surprising at first sight, the ATS function is not considered safety critical, given that safety is built in at the level of the interlocking and ATP functions. However, the ATS function is a nerve center because of its central position in relation to operators' decisions and actions, especially in degraded situations where operator intervention is required. Indeed, the analysis of some railway accidents has shown that consequences would have been far less severe if operators had performed appropriate "last chance" recovery actions (but they did not do so through lack of information, or lack of time, etc.) For this reason, a recent tendency has been to assign to modern ATS subsystems a non-zero Safety Integrity Level (SIL is a measure of the degree of confidence in the safety of a function, level 0 indicating no safety constraint at all, and 
level 4 being the maximum value). In this perspective, the aim of our approach is to evaluate the impact of the ATS subsystem on the global safety of a railway system. Technical components having attained a high safety level, attention should now be focused on the evaluation of human-machine interactions and their impact on safety. This approach requires cooperation between railway engineers (for the technical component), human and social scientists (for the human and organizational component) and safety engineers (for the synthesis and the global evaluation of the safety level.)

To illustrate the interest of a systemic approach in this interdisciplinary context, we now present a case study using FRAM as a complement to classical safety analysis for a scenario involving the locking of a switch for the protection of maintenance agents on the track. In this example interactions between operators, as well as interactions between operators and machines, have a significant impact on safety.

\subsection{Case study}

The protection of maintenance teams on sections of track requires the locking of switches that otherwise might direct trains towards the working zone. Any failure of the locking operation could be fatal for the workers involved. The locking operation is performed in collaboration between the head of maintenance team and the ATS operator. It requires a direct control of the interlocking function (the "ultra safe" function making it impossible to set conflicting routes), which is in stark contrast to the usual mode of operation, given that most executable controls are normally "filtered" by the interlocking and ATP functions (an unsafe command issued by an ATS function will simply not be executed). Here the command is directly transmitted 
to safety installations, without validation by another "ultra safe" function. For this reason safety engineers have put in place a safety procedure called "High Integrity Level Commands" (HILC). This procedure is a sequence of safety related communications between ATS computer systems in OCCs and the protection system which controls the switches (interlocking). An error detection code based on a Cyclic Redundancy Check (CRC) ensures the integrity of digital data exchanges between computers. The operator is obliged to use a double command mechanism which protects against unintentional commands. The HILC sequence for locking a switch takes place as follows (see Figure 5):

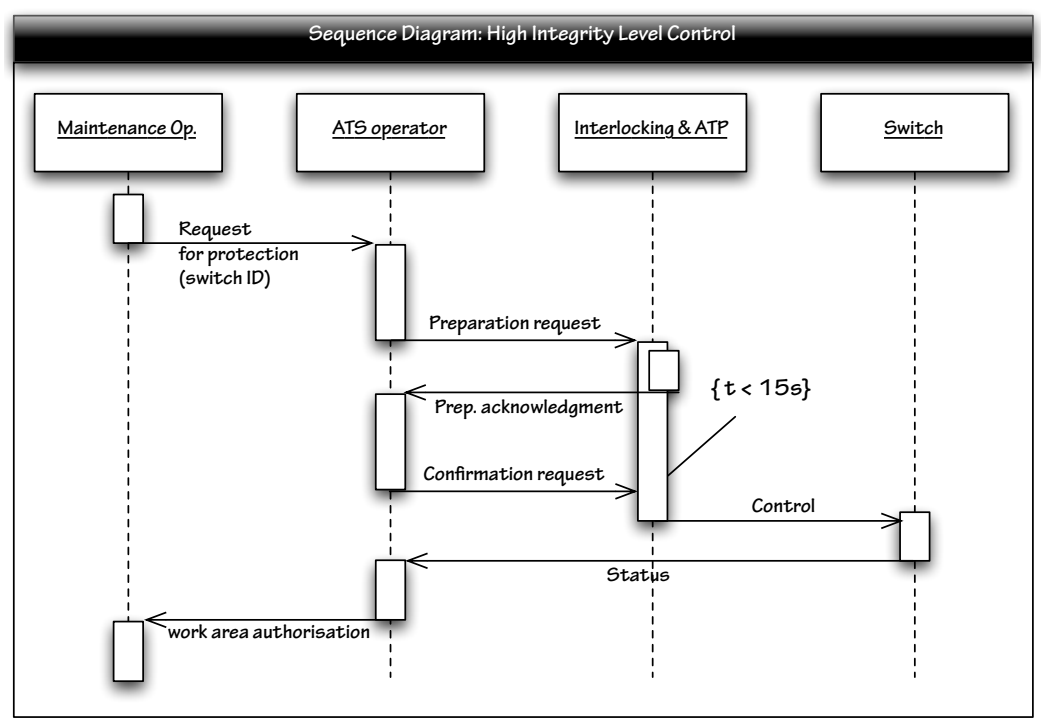

Figure 5: High Integrity Level Commands

1. The maintenance operator on the track looks for the identification number of the switch to lock and asks for protection from the ATS operator; 
2. The ATS operator sends a preparatory request to the protection system;

3. The protection system (control / monitoring of the interlocking function) sends an acknowledgement of this preparatory request to ATS;

4. After making sure that the received acknowledgement corresponds to the request, the ATS operator validates the request by sending a confirmation request;

5. The protection system ensures the validity of the message by checking the coherence between the CRCs of the two messages received from ATS, performs the locking of the correct switch, and then sends an acknowledgement to ATS indicating the status of the switch operation;

6. Finally, the ATS operator checks via a visual display that the switch is locked, and confirms this fact to the maintenance operator in the field.

\subsection{Safety Analysis}

\subsubsection{Classical safety analysis}

The potential for accidents is linked to the delay introduced by the ATS operator when seeking to detect uncompleted or erroneous HILCs. Safety engineers build fault trees based on functional analysis for each potential accident, having previously identified potential hazards. This analytical technique is based on a linear accident model in a discrete state space of the system. The fault tree analysis of a potential "failure of switch locking" accident is shown in Figure 6. Rectangles represent "function failure" events. Logical gates indicate the conjunction or disjunction of lower level failures resulting in a given upper level failure. Circles indicate that the event will not be further decomposed and that a given occurrence rate can be attributed 


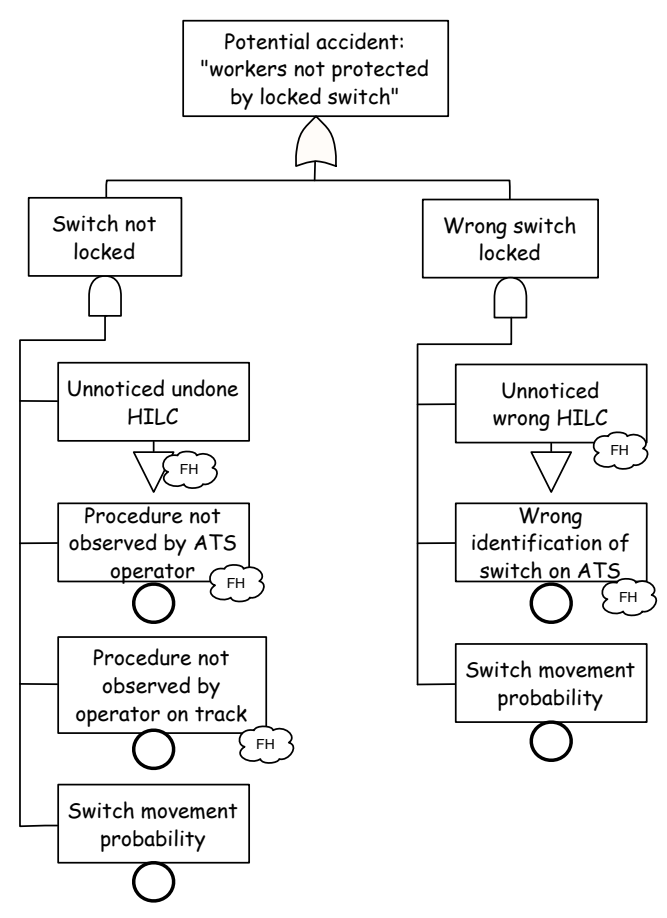

Figure 6: Fault Tree for "Potential accident by failure of switch locking"

to it. Inverted triangles, on the other hand, mean that the event is decomposed into other elementary events shown in another fault tree. For a better readability, the label "FH" identifies events that are due to human factors.

Probability values for pieces of technical hardware are determined based on studies of the behavior of specific components, on experience feedback or using data from suppliers. Probabilities of failure of actions by operators are generally taken from the human reliability literature, for example, work by Swain [39] and Rasmussen [40]. The likelihood that an operator does not respect a procedure will depend on contextual factors including experience and training, and these are taken into account. Typically, for an adequately trained operator performing a habitual activity, a failure probability of $10^{-3}$ 
will be used.

The result of this method applied to the case of an HILC switch locking a maintenance zone shows that a potential for accident could arise from the joint failure of at least three operations, with a failure probability of less than $10^{-3}$ for each operation.

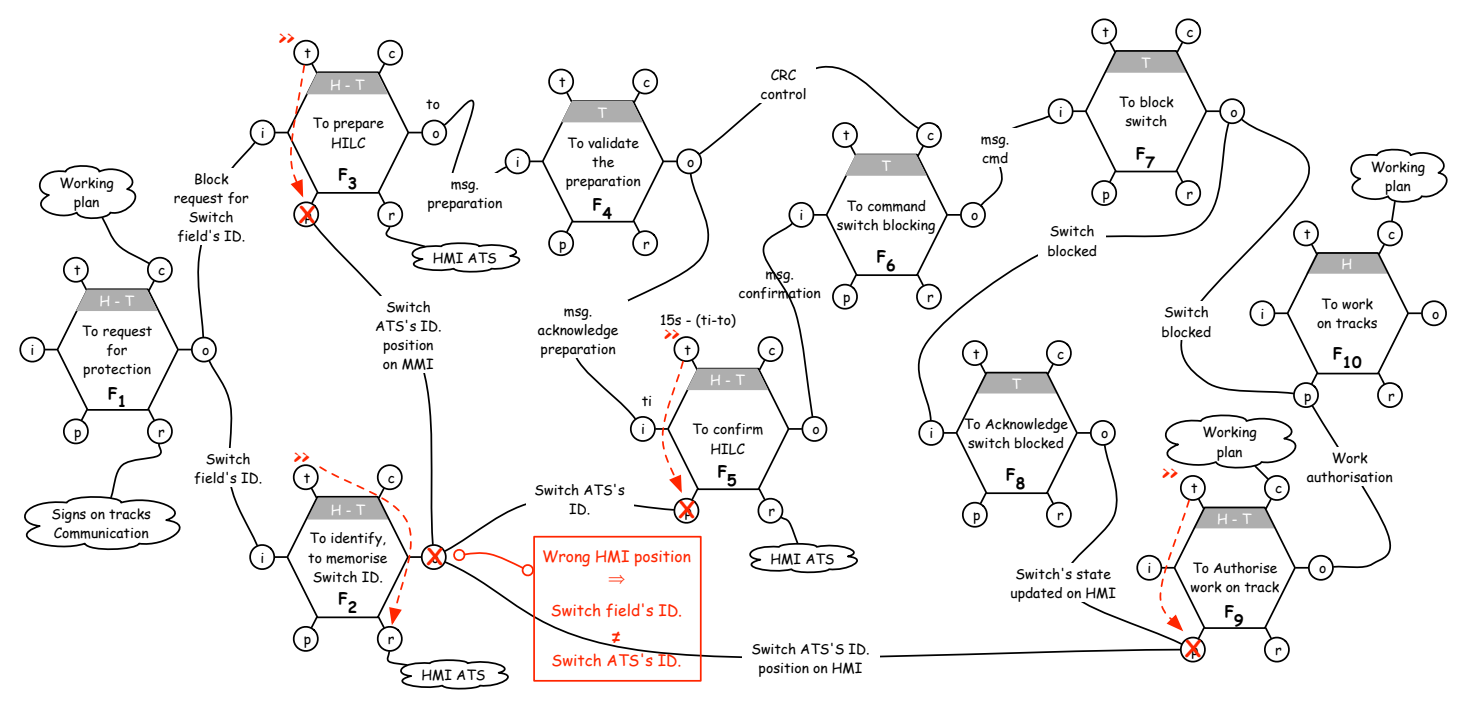

Figure 7: Degraded FRAM network

\subsubsection{Complementary approach using FRAM}

The FRAM network of an HILC switch locking a maintenance zone is shown in Figure 4. The maintenance operator, requesting protection for a particular working section, initiates the procedure. Two pieces of data are used by this procedure: the locking request and the identification number of the concerned switch. These two data are sent to the ATS operator. In the figure, this is expressed by two functional relations between the maintenance operator's activity and the ATS operator's activity, first in identifying the 
correct switch via the ATS interface, and secondly in preparing the HILC protection.

The procedure mainly requests actions by human operators. It is therefore possible to visualize many scenarios, simply by modifying the variability of performance of the operational units performed by the ATS operator.

As an example, Figure 7 shows a scenario in which the ATS operator's performance condition, in relation to the available time, is unsuitable or unpredictable. In the diagram this time pressure is represented by the symbol ( ») next to the time attribute of actions performed by this operator (the notation used in the example described in [16]). This pressure can be explained by the urgency of the situation or by an excessive number of objectives that the operator is attempting to meet. Thus, the operator does not correctly identify the switch to lock, the time pressure being too great to correctly execute control operations requested for identification via the ATS human-machine interface. This is represented by the cross next to the connectors of the function, which is dependent on the switch identification activity. The negative impact associated with the time pressure restricts the precondition for the operator's next activity, which means that the two acknowledgements sent by the control-command protection system are not checked, and the switch locking is not confirmed to the maintenance operator. The outcome is that an HILC for locking a switch has been executed, but for the wrong switch, and so the maintenance operators are not protected.

FRAM enables fault tree events to be analyzed very thoroughly. The example just described allowed a deeper analysis of the human causes of the "wrong switch locked" event than is possible in classical fault tree analysis 
(see Figure 6). The main contribution of FRAM is that it highlights complex interactions that arise in the socio-technical system and which provide the biggest clues in identifying the pertinence of the scenarios.

\subsection{Interdisciplinary Study}

Other scenarios can be described using a similar model. This kind of model is currently used as a common support between safety specialists and human and social scientists specializing in cognitive ergonomics. To calibrate the model (i.e. identify the potential variability of each functional unit) it is possible to use feedback data from actual experience, as well as the results of experiments performed in the laboratory.

This approach will be illustrated by a second case study concerning railway traffic supervision of an OCC's ATS functions. The aim of this activity is to monitor traffic using information provided by the ATS human-machine interface, to detect any incidents (especially those with an impact on safety), to diagnose them and to perform appropriate recovery actions. The FRAM network proposed for this activity is shown in Figure 8. The different operational units are the monitoring strategy of the Schematic Control Panel (SCP) which provides the global overview of the traffic, traffic monitoring, incident detection, incident diagnosis, selecting a recovery action, and finally the action itself. The FRAM network highlights the links between these functional units which can generate the Functional Resonances. Figure 8 shows the normal situation where basic input-output dependencies between functional units are obvious. Notice the time dependency of the detection activity on the monitoring strategy. This link indicates that the time needed to detect an incident depends on the strategy chosen for monitoring. To 
calibrate this model, an experimental ATS platform called SPICA-RAIL was installed in the Heudiasyc Laboratory at Compiègne University of Technology [41]. This platform is used as a support for studying the behavior of ATS operators. Using simulated railway traffic, the platform can reproduce the main aspects of OCC activities. On the one hand the FRAM analysis lets researchers consider various hypotheses regarding operators' performance conditions and how these might impact safety, and on the other hand it can synthesize results into scenarios for use in classical safety analysis. These scenarios may then be validated by experiments performed using the SpICA-RAIL platform. A first series of experiments was conducted, aiming to validate an experimental protocol and to calibrate the FRAM analysis.

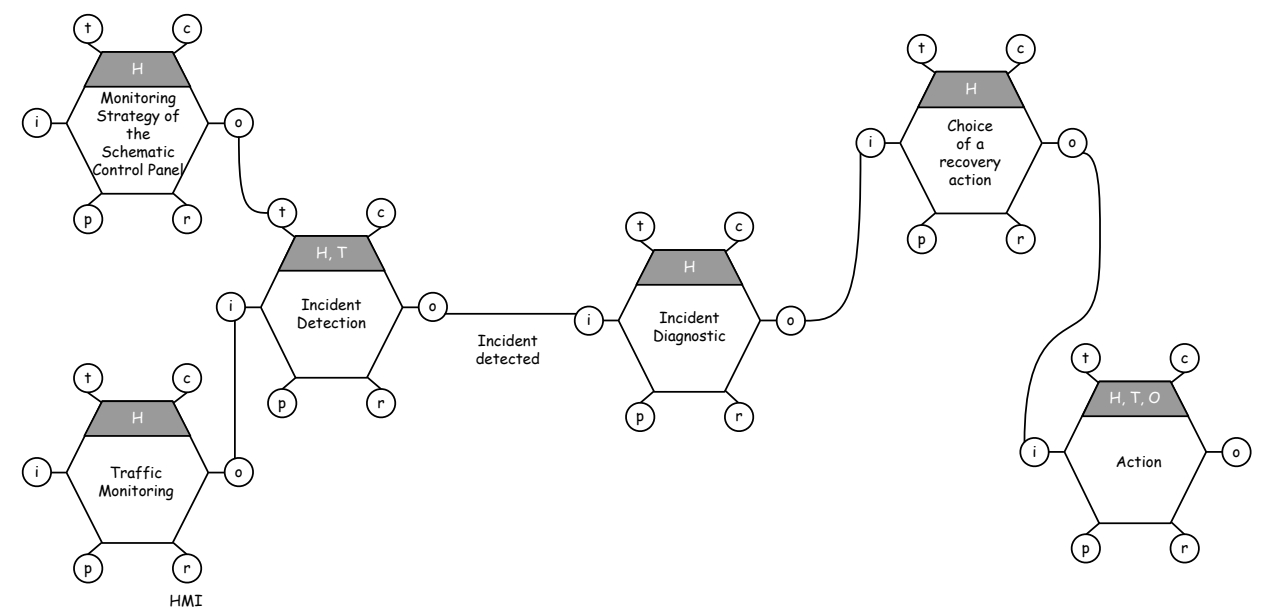

Figure 8: FRAM network of incident detection and recovery 


\subsection{Experiments}

\subsubsection{SPICA-RAIL platform}

SpICA-RAIL is based on a real Automatic Train Supervision (ATS) product developed by Alstom Transport. The ATS system is connected to the interlocking system and the Automatic Train Control (ATC) system (that includes Automatic Train Operation and Automatic Train Protection; see [42] for detailed descriptions of these systems). The ATS performs a broad range of traffic supervisory functions, from staff and rolling stock management to the monitoring and control of signaling and route setting. Signaling and route setting, intensively automated over the last decade, remain safety critical operations when degraded circumstances occur [43]. Interlocking and ATC are simulated in SPICA-RAIL by the traffic simulator system developed by Alstom Transport for validating and testing ATS projects. The traffic simulator simulates railway traffic operations through the use of a scripting language.

The ATS configuration delivered by Alstom for the SPICA-RAIL platform is the clone of a recent project. The line supervised is twenty-one kilometers long, with two tracks (one for each direction of travel in nominal operation) and a suburban traffic profile. It includes a branching line, commercial stations and several sets of interline points (allowing temporary single-track operation in case of failure, which includes failures affecting a particular zone on one track). This track plan is a typical suburban railway line operating homogeneous trains (same speed, same size, same weight). This kind of traffic is generally regulated by the frequency interval between trains (a regulation policy termed "constant headway" aiming to maintain as regular an interval 


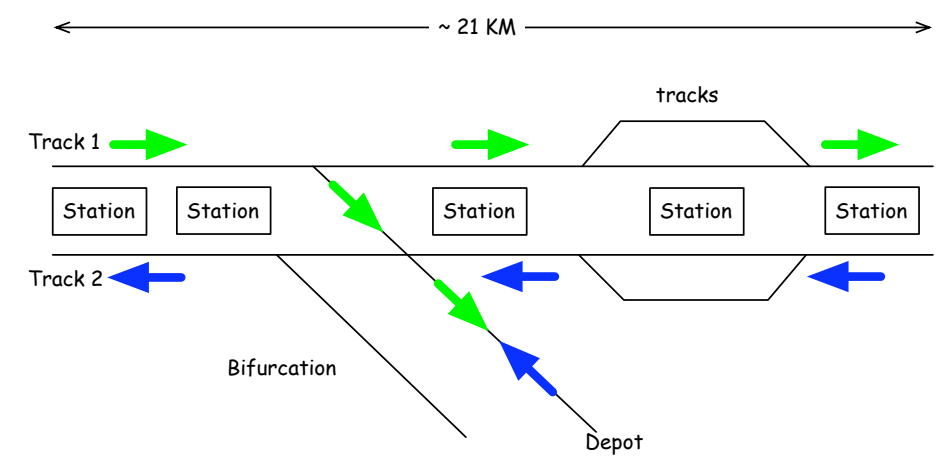

Figure 9: SPICA-RAIL track plan

as possible between successive trains). In order to generalize our experiments to main railway lines (operated with different types of trains with different speeds, sizes and weights, making it essential for trains to be able to overtake one another), the initial SPICA-RAIL network configuration was modified by the addition of passing tracks at a particular station. The regulation policy of this second kind of network is termed "time schedule" (aiming to maintain the schedule of each train as closely as possible). A simplified view of the modified track plan is provided in Figure 9.

The human-machine interface is composed of two elements. The first is a general MIMIC display known as the Schematic Control Panel (SCP) that can be seen by every operator in the OCC. The second element corresponds to operator workstations ("supervision computers") which give different views of the railway track plan. Figure 10 shows the SPICA-RAIL platform and the general HMI of the track overview. Figure 11 shows the specific signaling elements of the SCP. 

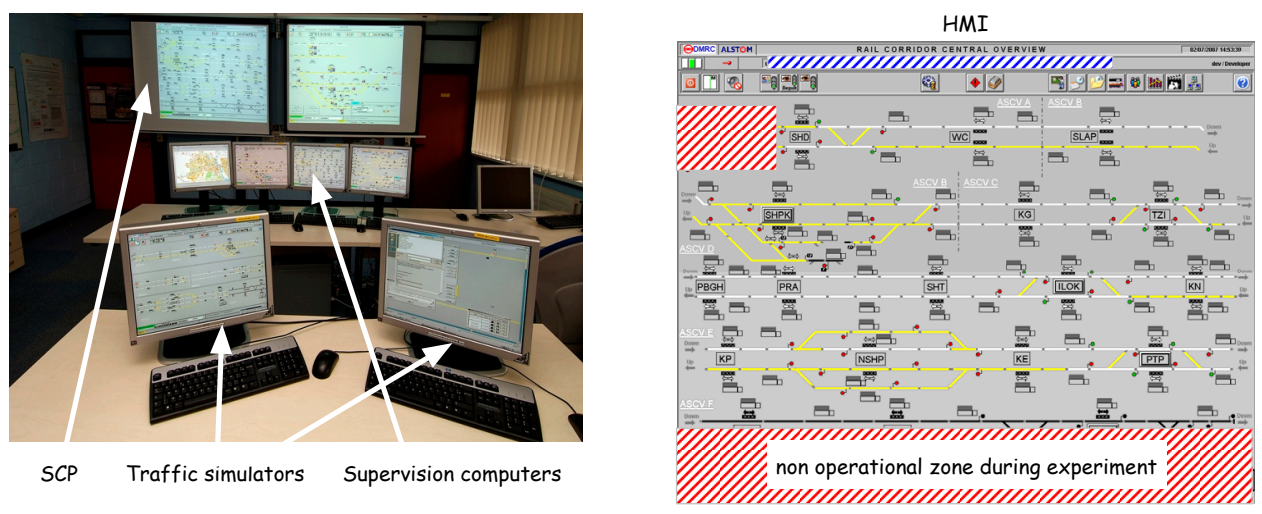

Figure 10: SPICA-RAIL platform

\subsubsection{Experimental protocol}

The protocol was defined in order to evaluate the performance of the graphical view that exists to provide useful information to the human operators. During evaluation the integrated alarm system was disconnected from the ATS.

Three experimental subjects (post-doctoral students in computer science assuming the role of ATS operators undergoing training) participated in the experiments. For practical reasons, it was not possible to perform experiments with more subjects because of the considerable amount of data and videos to process. This will be done in subsequent work. However, significant results were obtained. Prior to the experiment, each of them was trained to supervise nominal situations and to detect three representative types of incidents:

- Uncontrolled points. This problem can arise from a failure of the position sensor that renders it unable to indicate the current position of the points. The signal protecting the points therefore remains blocked 


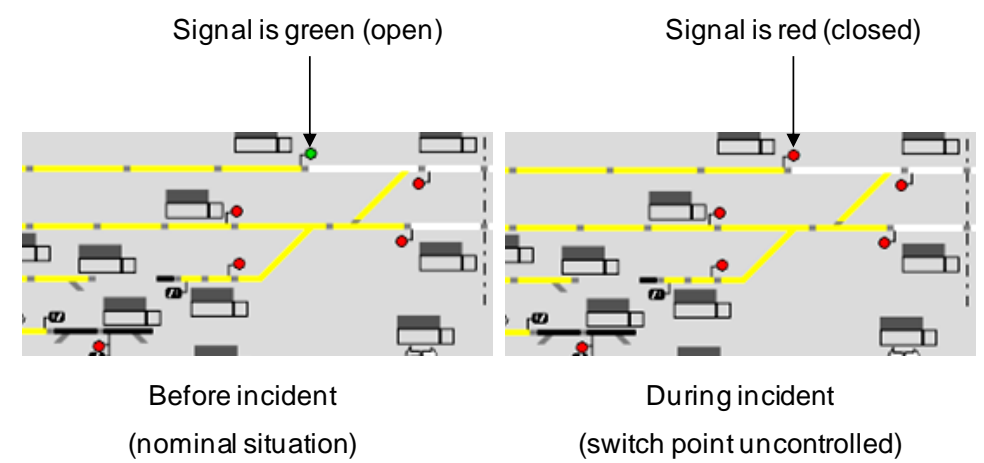

Figure 11: SPICA-RAIL signaling elements

at absolute stop. This incident is not safety critical, but entails serious disturbances to the traffic, and throttles activity as a result of degraded operating modes such as temporary single track. Figure 11 illustrates scenarios where an "uncontrolled points" incident occurs on the HMI;

- Signal failed to open. This might be a consequence of the previous type of incident, but it can also arise out of failures of the signal itself or of devices controlling it (in the latter case there are fewer visible symptoms to help diagnose the initial cause of the incident). This incident is not safety critical, but like the previous type of incident it can seriously impact network traffic;

- Signal failed to close. This is of course a safety-critical incident, whose consequences, including collisions and derailments, are potentially catastrophic. A possible cause would be a failure in the control-command devices which, although highly improbable (because of a "fail safe" design), is nevertheless imaginable. Given the ease with which this very 
exceptional situation can be simulated using the experimental platform, it was judged interesting to include it in the experimental protocol (an adapted reaction on the part of operators having the potential to reduce dramatically the severity of consequences).

Four independent variables (i.e., under the experimenter's control) were included in the protocol in order to evaluate the monitoring efficiency of the experimental subjects in different traffic supervision configurations. The dependent variable (i.e. the variable providing an assessment of subjects' performance) was the incident detection time (in seconds). Using the FRAM analysis, hypotheses were formulated regarding the influence of each of these parameters, and the experimental sessions aimed to validate (or invalidate) these hypotheses.

- The first variable was the type of incident (uncontrolled points vs. signal failure). Because uncontrolled points generate more indices on the HMI, the hypothesis for this variable was that detection time would be lower for this type of incident than for the two cases of signal failure. It was also expected that a signal failing to close would be more efficiently detected than a signal failing to open, because of the seriousness of potential consequences in the former case;

- The second variable was the time at which the incident occurred in relation to the duration of the supervision session (i.e. early or late in the session). For the purposes of simplification each scenario was 30 minutes long. The hypothesis regarding this variable was that detection would be faster at the beginning of the session, because of the monotony 
associated with a long period of nominal operation;

- The third variable, dependency, corresponded to the presence or absence of a causal relation between the incident and subsequent train behavior (no dependency meaning that the incident had no impact on trains, and dependency that the incident caused a train to stop). For this variable, the hypothesis was that human operators would periodically focus their attention on dynamic elements of the HMI. Consequently, detection should be easier when trains were circulating in the vicinity of the incident;

- The fourth variable was the type of setting for the route affected by the incident (permanent vs. non permanent). Routes are track sections (generally between two sets of points) where trains are authorized to circulate. There are two ways of setting a route in ATS: a permanent route is set once and not cleared by a train running along this route (so remaining set for the next train) whereas an automatic destruction route is specifically set for one train and cleared after the train has run along the route. Routes running through stations are generally set in permanent mode, while the automatic destruction setting is used for routes going to "passing tracks" (places where another train may overtake). Because there are more graphical indices in cases of equipment failure on permanent routes, the hypothesis for this variable was that detection times should be shorter for permanent routes.

Among the twenty-four possible types of scenario (corresponding to the number of combinations of values for the four variables) twenty could be 
implemented on the platform. For each one, a specific scenario was created. Ten "distraction" scenarios were also introduced in order to prevent subjects making inferences about sequences of different types of incidents.

The complete session of 30 scenarios was performed for each of the three experimental subjects, who were asked to indicate and explain orally each failure as soon as they detected it. In addition to the detection time and number of correct/incorrect detections (i.e., dependent variables), subjects' actions and verbalizations were video-recorded.

\subsection{Results}

Ten hours of recorded video per subject were collected. The great majority of equipment failures were detected. The rates of non-detection and false detection were about $1 / 30$ for each subject. Given that the subjects had only just been initiated into railway supervision, these results show that the training received was effective.

Because of high inter- and intra-subject variability and the presence of extreme values, means and variances could not be used. A statistical analysis was therefore performed using medians and ranges. The median detection time $\left(T_{D}\right)$ was $11,94 \mathrm{~s}$ with a range of $299 \mathrm{~s}$. Analysis of detection times based on test scenarios reveal a high variability between subjects. Medians and ranges for the three subjects are presented in Table 2 .

It might first be remarked that detection times are relatively high, considering the favorable experimental environment compared to the real environment:

- Subjects know that there is one failure to be detected in each scenario (this is not the case in real situations); 


\begin{tabular}{|c|c|c|}
\hline Subject & $T_{D}$ median (sec.) & Range \\
\hline 1 & 5,48 & 299 \\
\hline 2 & 22,42 & 160,9 \\
\hline 3 & 11,57 & 298,96 \\
\hline
\end{tabular}

Table 2: Detection times: Medians and ranges

- There are only three possible types of failure. In real situations there will be a greater variety of possible events;

- The duration of scenarios was very short $(30 \mathrm{mn})$ compared to the 6-hour shifts undertaken by real OCC operators;

- Failure detection was the only task to be performed, whereas in ATS several activities may be performed in parallel (for example, the manual setting of some routes);

- The track plan was reduced to a short zone with a single branching.

Secondly, it will be observed that subject number two appears noticeably less efficient (in terms of the detection time for incidents) than the two other subjects. $60 \%$ of subject number two's detection times exceed $20 \mathrm{~s}$. The rate is only $20 \%$ for subjects one and three. This results suggests that detection time is highly dependent on the strategy used by operators to explore the HMI and that this strategy is not the same from one individual to another. Moreover, the failure information given by HMI are not sufficiently striking to be perceived in the same manner by all subjects. In order to confirm the significance of this difference, statistical tests were performed on the rough results. 
The data collected does not satisfy normality and homogeneity conditions, and consequently the statistical significance of every comparison between modalities of subjects and variables was evaluated using non-parametric testindependent observations. The significance threshold was fixed at $\alpha=0,05$ for each comparison.

The Mann-Witney test for independent groups was used to compare subjects. This statistic takes account of the total number of observations made by the first subject that are greater than observations made by the second. Table 3 shows the p-values, that represent the probabilities of false rejections on the null hypothesis that the two samples are identical.

\begin{tabular}{|c|c|c|}
\hline Subjects & $p$ & $H_{0}: T_{D}^{1}=T_{D}^{2}$ \\
\hline Subject 1 - Subject 2 & 0,00041 & Significant \\
\hline Subject 1 - Subject 3 & 0,21 & Non significant \\
\hline Subject 2 - Subject 3 & 0,03 & Significant \\
\hline
\end{tabular}

Table 3: Comparison between subjects: Mann-Witney, $\alpha=0.05$

This result confirms that detection times for subject number 2 are indeed significantly different from the two others.

In order to evaluate the impact of the traffic configuration variables on the detection times, the Wilcoxon signed-rank test for paired comparisons was used. This test is usually used for two related samples or repeated measurements on a single sample. It involves comparisons of differences between measurements.

Unexpectedly, only one comparison reached significance. Sample size seems to be responsible for this lack of significance. Nevertheless, a nonparametric paired rank test such as the Wilcoxon test may be applied to 
these data. In every case the number of pairs is greater than 8 . This kind of test could, moreover, give results for fewer pairs [44].

However, the effect of the dependency variable denoting the presence of trains in the vicinity of the incident produced a significant result. It was assumed that the detection time would be lower when a train was nearby. In fact, the Wilcoxon statistic reveals the opposite effect, and with significance. This result is quite surprising. It seems reasonable to suppose that the strategy used by the human operator would be to follow periodically the movements of trains. Complementary investigations should be performed to shed further light on this effect.

\subsection{Analysis}

The performance conditions investigated in this first series of experiments concerned time pressure. The time taken by operators to detect abnormal events was measured. The first results suggest that in a simplified simulation environment where the detection of abnormal situations arguably presents fewer challenges than in the real world, some subjects nevertheless fail to detect these abnormal situations at all quickly. Placed in the framework of FRAM, these first elements enable an order of magnitude to be assigned to operators' performance in a particular context.

Experimental results also confirm differences between strategies employed by human operators, using the human-machine interface under investigation, to detect equipment failures impacting traffic safety. Clearly, not all operators use the same strategy, which creates a wide spread of varying performances. The reasons for these differences need to be explored through a more detailed analysis of operators' behavior. 
However, merely knowing that there is a dispersion of monitoring strategies which impacts incident detection enables us to identify a potential scenario of inadequate decision as a consequence of a "functional resonance" phenomenon. Figure 12 illustrates such a scenario, and show how high variability propagates within the FRAM network modeling operator's activity. An inadequate monitoring strategy leads to late incident detection, which in turn leads to time pressure on the diagnostic operational unit. In nominal situations this operational unit makes adequate decisions (no problems of diagnosis came to light during our experimental sessions) but in other situations time pressure could lead to incorrect choices of recovery actions, especially where a situation is safety-critical and where there is little time available to avert an impending accident. An inappropriate recovery action means that the ATS operator might not succeed in reducing the gravity of an accident.

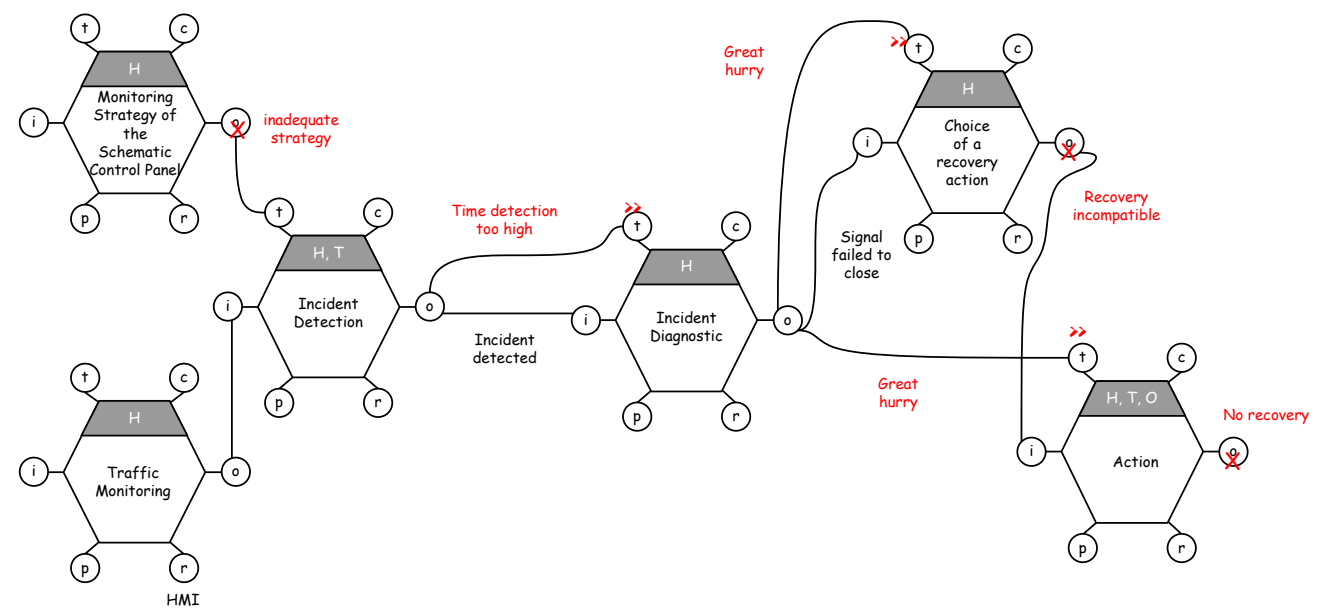

Figure 12: FRAM scenario model of incident detection and recovery 
Other experimental series will therefore be performed using the SPICARAIL platform and the standard model presented in this paper, using also oculometers to record operators' eye movements. Results obtained should help to clarify the reasons for the dispersion of performances, and possibly to identify new accidental scenarios.

\subsection{Proposals for ergonomic enhancements}

The experimental results presented above highlight the importance of ATS interfaces in the efficient detection of incidents and, like certain previous

studies [45, 46, 47], suggest that ATS interfaces could be better adapted to operator's needs than they currently are.

Armed with the results of the present study and with research findings in the literature, a specific working group was therefore constituted by Alstom to draw whatever conclusions may be drawn and to rough out some proposals for ergonomic requirements for ATS HMI that might enhance the performance of the joint ATS-operator system [34].

These proposals are listed below:

- To reduce the number of graphical elements to what is really needed by the operators:

- For any safety-related operational situation, the HMI should display "just enough" (i.e. necessary and sufficient [48]) information to enable a safe situation to be maintained or recovered. Too much information makes the important information less salient and increases the operator's cognitive load [46]; 
- For other operations, the HMI should show only information required to anticipate future events:

* In nominal situations (automatic mode), the ATS HMI should allow the operator to anticipate degraded modes (in realistic situations operators prefer to adopt an anticipative than a reactive mode [46]);

* In degraded situations (manual mode: operations are performed by the operator):

- The ATS HMI should allow the operator to predict what might happen if no action is performed;

- The ATS HMI should allow the operator to anticipate the result of any action (for example, the result of a "cancel route" action will stop a specific train at a signal within $\mathrm{x}$ seconds and begin to disrupt regular traffic within $\mathrm{y}$ seconds);

- Analogue representations should be preferred to digital displays: digital information need to be computed/interpreted by the ATS operator, whereas analogue information provides a more intuitive representation. Moreover, analogue representations enable the use of various perceived affordances [49];

- The HMI dynamics should be adapted in real time to mental activity required by the operators (the decision process is continuous and needs to be linked to the dynamic situation [48]);

- The response time of the supervised equipment in the field should be 
real-time constrained and certified (response time is a crucial parameter in dynamic situation supervision $[50,51])$;

- Operators should at all times know which control systems are participating their Safety Integrity Level. In particular, operators shall not have over-confidence in system safety;

- In case of simultaneous incidents, the HMI should focus the attention of the operators on the most severe safety-critical event, such as "signal failed to close" or "signal passed at danger". The operators' attention should be drawn to the specific part of the interface where the event has occurred through the aid of a specific visual alarm, instead of via a textual alarm on the general interface without a precise location.

These proposals need to be tested in experimental, simulated or realistic situations before being adopted as guidelines for future ATS systems.

\section{Conclusion}

The interest of a systemic approach is to offer a complementary exchange support between risk management and human and organizational factors. Indeed, analysis of accidents involving human and organizational factors clearly demonstrate that methods using a sequential accident model are not sufficient. In this perspective, the FRAM method enables experimental results from cognitive psychology and from classical safety analysis to be integrated using the same formalism. Used as a complement to classical safety analysis, the FRAM method explores the impact of human and organizational factors more thoroughly than a simple allocation of error probabilities is able 
to achieve. Moreover, a dynamic representation of the system using qualitative attributes can identify new scenarios for incidents or accidents, and these can be analyzed via new hypotheses that can be tested in the field or in a simulated environment. Whereas classical safety analysis methods show elements that are going wrong, FRAM also allow the execution of functions or activities that are performing correctly [16]. To this extent, classical methods and FRAM analysis are complementary, and their combined use in a systemic approach is a step towards a better understanding the activity of real operators, taking into account psychological and ergonomic factors. In this perspective, a fruitful improvement of FRAM method would be to include quantitative aspects allowing the relative likelihood of the different scenarios to be estimated using belief functions. This is the subject of subsequent work aiming at assigning likelihoods to each functional unit input and propagating them in the FRAM network.

The systemic approach used in this present study has already provided fruitful results in aeronautic and industrial supervision situations, and more recently in railway traffic control [46]. The results presented in the present paper confirm the pertinence of this approach for a better understanding of the determining parameters for a complex socio-technical system such a railway Operating and Control Centre. They show that even when given identical training, different operators do not use the same monitoring strategy, and the consequence is a very high variability in the time taken to detect incidents (variability in strategies used by operators to control railway traffic has already been observed in naturalistic and simulation-based studies; see for instance [52] and [47]). These strategies should be described in more 
details, and this is the subject of a study in progress, but for the moment the key point is to improve the ergonomics of the ATS interface in order to reduce this variability. Some proposals by a specific working group have been presented in this paper, and their efficiency will be tested in future experimental sessions. The determining factor is to provide the operator with "just enough" information for a clear understanding the present situation and for anticipating the immediate future. This goes through an adapted "pre-diagnostic" performed by automatic systems, the final diagnostic always remaining, at least in degraded situations, a human prerogative. In a broader perspective, it will be necessary in the future to define and design the right level of cooperation between more and more automated machines and human operators $[53,54]$.

\section{References}

[1] R. E. Barlow, F. Proschan, Mathematical theory of reliability, Wiley, New York, 1965.

[2] A. Villemeur, Reliability, availability, maintainability, and safety assessment, J. Wiley, Chichester, 1992.

[3] IEC, 61508:1998 and 2000, part 1 to 7. Functional Safety of Electrical, Electronic and Programmable Electronic Systems., 2000.

[4] CENELEC, EN-50126: Application ferroviaires - Spécification et démonstration de Fiabilité, Disponibilité, Maintenabilité et Sécurité (FMDS), Norme, CENELEC, 1999. 
[5] CENELEC, EN-50128: Applications ferroviaires - Système de signalisation, de télécommunication et de traitement - Logiciels pour systèmes de commande et de protection ferroviaire, Norme, CENELEC, 2001.

[6] CENELEC, EN-50129: Application ferroviaires - Système de signalisation, de télécommunication et de traitement - Systèmes électroniques relatifs à la sécurité pour la signalisation, Norme, CENELEC, 2001.

[7] N. Wiener, Cybernetics, Second Edition: or the Control and Communication in the Animal and the Machine, The MIT Press, 1965.

[8] E. M. Dougherty, Human reliability analysis - where shouldst thou turn?, Reliability Engineering \& System Safety 29 (1990) 283-299.

[9] E. Hollnagel, Cognitive reliability and error analysis method, Elsevier Science Ltd, Oxford, 1998.

[10] E. M. Dougherty, Is human failure a stochastic process?, Reliability Engineering \& System Safety 55 (1997) 209-215.

[11] P. Le Bot, Human reliability data, human error and accident modelsillustration through the three mile island accident analysis, Reliability Engineering \& System Safety 83 (2004) 153-167.

[12] A. Isaac, S. T. Shorrock, B. Kirwan, Human error in european air traffic management: the hera project, Reliability Engineering \& System Safety 75 (2002) 257-272.

[13] C. Bieder, Les facteurs humains dans la gestion des risques, évolution de la pensée et des outils, Hermès, Paris, 2006. 
[14] I. S. Kim, Human reliability analysis in the man-machine interface design review, Annals of Nuclear Energy 28 (2001) 1069-1081.

[15] J.-M. Hoc, Human and automation: A matter of cooperation, in: HUMAN'07, Timimoun, Algeria.

[16] E. Hollnagel, Barrier analysis and accident prevention, Aldershot, UK: Ashgate, 2004.

[17] F. Belmonte, Impact des postes centraux de supervision de trafic ferroviaire sur la sécurité, Ph.D. thesis, Université de Technologie de Compiègne, 2008.

[18] N. Limnios, Arbres de défaillances, deuxième édition revue et augmentée, Hermès Science, Lavoisier, 2005.

[19] N. Leveson, A new accident model for engineering safer systems, Safety Science 42 (2004) pp. 237-270.

[20] K. Vicente, Cognitive engineering research at riso from 1962-1979, in: E. Salas (Ed.), Advances in Human Performance and Cognitive Engineering Research, volume 1, Elsevier, New York, 2001, pp. 1-57.

[21] E. Hollnagel, D. Woods, Cognitive systems engineering: New wine in new bottles, International Journal of Man-Machine Studies 18 (1983) 583-591.

[22] D. A. Norman, Cognitive engineering, in: D. A. Norman, S. W.Draper (Eds.), User centered design: New perspectives on Human-Computer 
Interaction, Lawrence Erlbaum Associates, Hillsdale, NJ, USA, 1986, pp. 31-61.

[23] D. D. Woods, E. M. Roth, Cognitive engineering: Human problem solving with tools, Human Factors 30 (1988) 415-430.

[24] M. R. Endsley, R. Hoffman, D. Kaber, E. Roth, Cognitive engineering and decision making: An overview and future course, Journal of Cognitive Engineering and Decision Making 1 (2007) 1-21.

[25] L. G. Militello, C. O. Domingez, G. Lintern, G. Klein, The role of cognitive systems engineering in the systems engineering design process, Systems Engineering (2009) 1-12.

[26] J.-M. Hoc, F. Darses, Introduction, in: J.-M. Hoc, F. Darses (Eds.), Psychologie ergonomique : tendances actuelles, Presses Universitaires de France, Paris, 2004, pp. 1-13.

[27] M. D. Byrne, Cognitive architecture, in: A. Sears, J. Jacko (Eds.), The human computer interaction handbook: Fundamentals evolving technologies and emerging applications (2nd ed), CRC Press, Boca Raton, FL, USA, 2008, pp. 93-113.

[28] R. W. Proctor, K.-P. L. Vu, Human information processing: An overview for human-computer interaction, in: A. Sears, J. Jacko (Eds.), The human computer interaction handbook: Fundamentals evolving technologies and emerging applications (2nd ed), CRC Press, Boca Raton, FL, USA, 2008, pp. 43-60. 
[29] E. Hollnagel, D. Woods, N. Leveson, Resilience Engineering: Concepts and precepts, Ashgate, Aldershot, 2006.

[30] J. Reason, Human Error, Cambridge University Press, 1990.

[31] D. D. Woods, On taking human performance seriously in risk analysis: Comments on dougherty, Reliability Engineering \& System Safety 29 (1990) 375-381.

[32] C. W. Johnson, Failure in safety-critical systems: A handbook of accident and incident reporting, University of Glasgow Press, Glasgow, Scotland, 2003.

[33] D. D. Woods, E. M. Roth, K. B. Bennett, Explorations in joint humanmachine cognitive systems, in: S. P. Robertson, W. W. Zachary, J. B. Black (Eds.), Cognition, computing, and cooperation, Ablex Publishing Corp., Norwood, NJ, USA, 1990, pp. 123-158.

[34] D. Woods, E. Hollnagel, Joint Cognitive Systems, CRC Press, Inc., Boca Raton, FL, USA, 2006.

[35] E. Hollnagel, Dependability of joint human-computer systems, in: SAFECOMP '02: Proceedings of the 21st International Conference on Computer Safety, Reliability and Security, Springer-Verlag, London, UK, 2002, pp. 4-9.

[36] E. Hollnagel, D. Woods, Joint Cognitive Systems: Foundations of Cognitive Systems Engineering, CRC Press, 2005. 
[37] B. Johansson, Joint control in dynamic situations, Ph.D. thesis, Linköpings universitet, Institute of Technoloy, 2005.

[38] L. Bainbridge, Ironies of automation, Automatica vol. 19 (1983) 775779.

[39] A.-D. Swain, G. H-E., Handbook on Human Reliability Analysis with Emphasis on Nuclear Power Plant Application, Technical Report NUREG/CR-1278, USNRC, 1983.

[40] J. Rasmussen, Skills, rules, knowledge; signals, signs, and symbols, and other distinctions in human performance models, IEEE Transactions on Systems, Man and Cybernetics 13 (1983) 257-266.

[41] F. Belmonte, W. Schön, J. Boulanger, K. Berkani, Railway traffic supervision research program : Spica rail platform, in: EURNEX-ZEL, 14th international symposium "Toward the competitive rail systems in europe", Zilina, Rep. Slovaque (EU).

[42] F. Belmonte, K. Berkani, J. Boulanger, W. Schön, Safety enhancement of railway traffic by modern supervision systems, in: WCRR (Ed.), Seventh World Congress on Railway Research., Montreal (Canada).

[43] F. Belmonte, K. Berkani, J. Boulanger, W. Schön, Taking into account human factors in railway supervision, in: I. S. S. A. (ISSA) (Ed.), Ninth International Symposium of the ISSA Research Section: Design process and human factors integration: optimising compagny performance, Nice (France). 
[44] S. Siegel, N. Castellan, Nonparametric statistics for the behavioral sciences, McGraw-Hill, New York, 1988.

[45] S. Djibo, Analyse ergonomique et organisationnelle de la charge de travail des agents des postes de commandes centralisés (PCC) à la RATP, Technical Report, Report French PREDIT II research program, 2004.

[46] A. Kauppi, A human-computer interaction approach to train traffic control, Dissertation for the degree of licenciate of technology in computer science, Uppsala University, Sueden, 2006.

[47] C. Zanarelli, Caractérisation des stratégies instrumentales de gestion d'environnements dynamiques : analyse de l'activité de régulation du métro, Ph.D. thesis, Thèse de doctorat d'ergonomie non publiée. Paris : Université Paris 8, 2003.

[48] R. Amalberti, La conduite des systèmes à risques, Presses Universitaires de France, Paris, 2001.

[49] D. Norman, Affordances, conventions and design, Interaction VI.3 (1999) 38-42.

[50] J.-M. Hoc, Supervision et contrôle de processus : la cognition en situation dynamique, Presses Universitaires de Grenoble, Grenoble, 1996.

[51] J.-M. Hoc, R. Amalberti, J.-M. Cellier, V. Grosjean, Adaptation et gestion des risques en situation dynamique, in: Psychologie ergonomique : tendances actuelles, Presses Universitaires de France, Paris, 2004, pp. $15-48$. 
[52] T. M. J. Lenior, Analyses of cognitive processes in train traffic control, Ergonomics 36 (1993) $1361-1368$.

[53] D. Lenior, W. Janssen, M. Neerincx, K. Schreibers, Human-factors engineering for smart transport: Decision support for car drivers and train traffic controllers, Applied Ergonomics 37 (2006) 479 - 490.

[54] J. Wilson, B. J. Norris, Rail human factors: Past, present and future, Applied Ergonomics 36 (2005) 649 - 660. 